\title{
Novel Method of Quantifying Radioactive Cesium-Rich Microparticles (CsMPs) in the Environment from the Fukushima Daiichi Nuclear Power Plant
}

Ryohei Ikehara, $^{\dagger}$ Mizuki Suetake, $^{\dagger}$ Tatsuki Komiya, $^{\dagger}$ Genki Furuki, $^{\dagger}$ Asumi Ochiai, ${ }^{\dagger}$ Shinya Yamasaki, ${ }^{\dagger}$ William R. Bower, ${ }^{\S}$ Gareth T. W. Law, ${ }^{\S}$ Toshihiko Ohnuki, ${ }^{\|}$Bernd Grambow, ${ }^{\perp}$ Rodney C. Ewing, ${ }^{\#}$ and Satoshi Utsunomiya* $*+\infty$

\footnotetext{
${ }^{\dagger}$ Department of Chemistry, Kyushu University, 744 Motooka, Nishi-ku, Fukuoka 819-0395, Japan

${ }^{\ddagger}$ Faculty of Pure and Applied Sciences and Center for Research in Isotopes and Environmental Dynamics, University of Tsukuba, 1-1-1 Tennodai, Tsukuba, Ibaraki 305-8577, Japan

${ }^{\S}$ Centre for Radiochemistry Research, School of Chemistry, The University of Manchester, Oxford Road, Manchester, M13 9PL United Kingdom

"Laboratory for Advanced Nuclear Energy, Institute of Innovative Research, Tokyo Institute of Technology, 2-12-1 Ookayama, Meguro-ku, Tokyo 152-8550, Japan

${ }^{\perp}$ SUBATECH, IMT Atlantique, CNRS-IN2P3, The University of Nantes, Nantes 44307, France

\# Department of Geological Sciences and Center for International Security and Cooperation, Stanford University, Stanford, California 94305-2115, United States
}

Supporting Information

ABSTRACT: Highly radioactive cesium-rich microparticles (CsMPs) were released from the Fukushima Daiichi nuclear power plant (FDNPP) to the surrounding environment at an early stage of the nuclear disaster in March of 2011; however, the quantity of released CsMPs remains undetermined. Here, we report a novel method to quantify the number of CsMPs in surface soils at or around Fukushima and the fraction of radioactivity they contribute, which we call "quantification of CsMPs" (QCP) and is based on autoradiography. Here, photostimulated luminescence (PSL) is linearly correlated to the radioactivity of various microparticles, with a regression coefficient of 0.0523 becquerel/PSL/h $(\mathrm{Bq} / \mathrm{PSL} / \mathrm{h})$. In soil collected from Nagadoro, Fukushima, Japan, CsMPs were

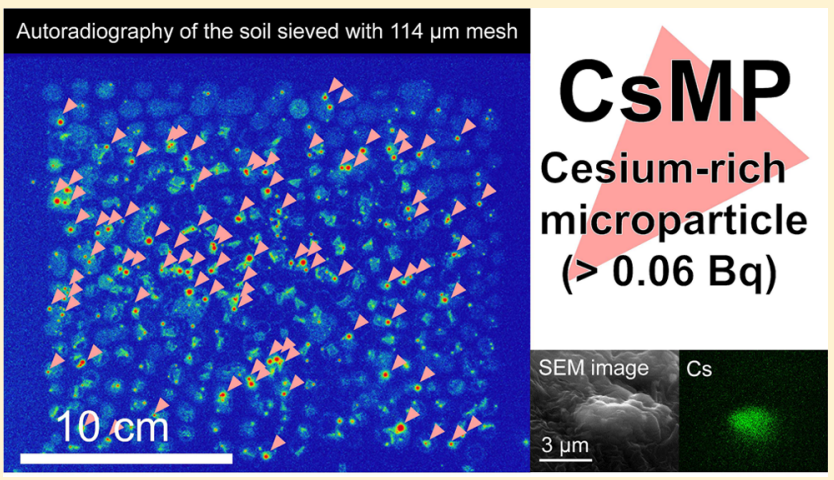
detected in soil sieved with a $114 \mu \mathrm{m}$ mesh. There was no overlap between the radioactivities of CsMPs and clay particles adsorbing Cs. Based on the distribution of radioactivity of CsMPs, the threshold radioactivity of CsMPs in the size fraction of $<114 \mu \mathrm{m}$ was determined to be $0.06 \mathrm{~Bq}$. Based on this method, the number and radioactivity fraction of CsMPs in four surface soils collected from the vicinity of the FDNPP were determined to be 48-318 particles per gram and 8.53-31.8\%, respectively. The QCP method is applicable to soils with a total radioactivity as high as $\sim 10^{6} \mathrm{~Bq} / \mathrm{kg}$. This novel method is critically important and can be used to quantitatively understand the distribution and migration of the highly radioactive CsMPs in near-surface environments surrounding Fukushima.

\section{INTRODUCTION}

The nuclear disaster that occurred after the great east Japan earthquake in March of 2011 caused serious damage to the nuclear reactor unit nos. 1-4 at the Fukushima Daiichi Nuclear Power Plant (FDNPP), ${ }^{1}$ which released a total of $\sim 10^{19} \mathrm{~Bq}$ of radioactivity associated with radionuclides such as ${ }^{133} \mathrm{Xe},{ }^{132} \mathrm{Te}$, ${ }^{131} \mathrm{I}$, and ${ }^{137} \mathrm{Cs}^{2}$ Of these radionuclides, $10^{15}-10^{16} \mathrm{~Bq}$ of ${ }^{137} \mathrm{Cs}$ was released to the atmosphere and dispersed to the surrounding environment. ${ }^{3-5}$ Even though the fraction of the released Cs was as much as $\sim 7 \%$ of the total inventory, ${ }^{3}$ radioactive $\mathrm{Cs}$ is currently responsible for nearly all the radiation dose in contaminated areas due to its half-lives of 2.065 years and 30.17 years for ${ }^{134} \mathrm{Cs}$ and ${ }^{137} \mathrm{Cs}$, respectively. ${ }^{6}$ The areas that were subject to Cs contamination span over 15 prefectures including the metropolitan city of Tokyo. ${ }^{7-10}$ The chemical forms of Cs released from the reactor were identified to be soluble hydroxides and chloride ${ }^{11}$ in addition to possibly

Received: December 29, 2017

Revised: $\quad$ May 11, 2018

Accepted: May 11, 2018

Published: May 21, 2018 
iodide, which was predicted in accident-code calculations ${ }^{12,13}$ and an experimental study. ${ }^{14}$ Kaneyasu et al. reported that the soluble form of Cs reacted with sulfate aerosol and was transported over long distances ( $>170 \mathrm{~km}$ from the FDNPP). ${ }^{15}$ The intrinsic forms of soluble Cs and the soluble Cs adsorbed onto the aerosols were then considered to have been deposited on the ground in the large area mentioned above by precipitation, primarily during the periods of March 15th and 16th in the northern Fukushima Prefecture and March 21st23rd in the Ibaraki, Chiba, Tochigi, and Saitama prefectures and Tokyo. 8,16

The deposited Cs subsequently dissolved in the meteoric water, and the hydrated Cs ions quickly adsorbed onto the soil forming minerals and reached adsorption equilibrium within $\sim 10$ days. ${ }^{17}$ The soluble Cs was efficiently adsorbed to the clay minerals due to its high affinity for edge and frayed-edge sites ${ }^{18-21}$ as well as organic matter. As such, the Cs remains in the surface soil within the top $5 \mathrm{~cm}$ of the vertical soil profile in Fukushima due to the tight binding between the $\mathrm{Cs}^{+}$, organic matter, and the mineral adsorption sites. ${ }^{6}$ Hereafter, clay minerals adsorbing Cs are referred to as "Cs-clay". Even though the radioactivities of ${ }^{134} \mathrm{Cs}$ and ${ }^{137} \mathrm{Cs}$ are as high as $\sim 10^{7} \mathrm{~Bq} / \mathrm{kg}$ in the soil in Fukushima, ${ }^{6}$ the Cs concentration is only at the level of $\sim 3 \times 10^{-1} \mathrm{ppb}$ due to dilution. Thus, FDNPP-derived Cs is barely detectable using microscopy techniques.

Conversely, several recent studies have characterized and reported a different form of Cs in the soils near the FDNPP. This sparingly soluble form of Cs comes in the form of Cs-rich microparticles (CsMPs), in which the Cs concentration is much higher $\left(\sim 0.55-30\right.$ wt $\left.\% \mathrm{Cs}_{2} \mathrm{O}\right)$, with the microparticles composed of amorphous $\mathrm{SiO}_{2}$ and $\mathrm{Zn}-\mathrm{Fe}$ oxide nanoparticles that are associated with $\mathrm{Cs}$ and even minor $\mathrm{U}$ and $\mathrm{Tc}{ }^{11,22}$ The nanoscale characteristics and isotopic signatures of these CsMPs provide many clues about the chemical reactions that occurred during the reactor meltdown at the FDNPP and provide a basis for understanding the properties of the debris. Their composition indicates an origin that is a mixture of melted nuclear fuels and structural materials. ${ }^{11,22,23}$ The CsMPs are very small, usually a few micrometers, and have an extremely high level of radioactivity, $\sim 10^{11} \mathrm{~Bq} / \mathrm{g}$, which, in natural water environments or in organisms, can produce high concentrations of hydroxyl radicals, such as $\bullet \mathrm{OH}$, by radiolysis within a thickness of a few hundred micrometers of the water layer adjacent to a CsMP. ${ }^{9}$ Due to the high production rate of hydroxyl radicals and the small size of the CsMPs, internal exposure to a micron-sized point source with extremely high radioactivity is potentially an important health concern.' Therefore, the distribution pattern of the amounts of CsMPs in the contaminated areas needs to be determined on a quantitative basis for better estimation of radiation exposure, especially in locations where residents have returned. Accordingly, in the present study, we focus on the quantification of CsMPs with a radioactivity density of $\sim 10^{11}$ $\mathrm{Bq} / \mathrm{g}$. This is explicitly different from another type of glassy particle bearing lower concentrations of Cs (undetectable by scanning electron microscopy-energy-dispersive X-ray analysis, SEM-EDX), in which the particle size range was much larger $(70-400 \mu \mathrm{m}) .^{24}$

The CsMPs can be imaged as localized radioactive spots using autoradiography of soil samples. ${ }^{25}$ In general, the radioactive spots are distributed heterogeneously within the broad distribution of a weak radioactive background that corresponds to the Cs-clay. The highly radioactive spots can be removed with adjacent soil particles using double-sided tape, and the subsequent $\gamma$ spectrometry can roughly estimate the radioactivity fraction derived from CsMPs within soil samples with known radioactivity. However, this procedure still involves large uncertainties and produces unreliable results for determining the fraction of radioactivity derived from CsMPs because many Cs-clay particles are also picked up by the double-sided tape, thus introducing large errors and uncertainties in the determination of Cs fraction. More importantly, the Cs-clays are also easily recorded as localized radioactive spots. ${ }^{26,27}$ Even though the adsorbed radioactive Cs is undetectable with electron microscopy at $<0.1 \mathrm{wt} \%$, the radioactivity of a large Cs-clay particle and large aggregates of fine clay particles can be high enough to appear as radioactive spots on imaging plates. ${ }^{28}$ While autoradiography can be used to estimate the radioactivity of individual radioactive particles from Chernobyl ${ }^{29}$ and Fukushima, ${ }^{30,31}$ its application to the quantification of CsMPs has never been explored. As reported in previous studies, individual CsMPs can be separated and characterized in detail using electron microscopy techniques and $\gamma$ spectrometry; ${ }^{11}$ however, it is impossible to separate and characterize all individual CsMPs in a given sample because the number of radioactive spots is too large to count and the efficiency of successful separation is not $100 \%$. Therefore, the development of an efficient and simplified method to quantify the number and radioactivity of CsMPs is important. Here, we describe a novel application and significant expansion of a classic measurement technique (autoradiography) to quantify the fraction of radioactivity contributed by the CsMPs in contaminated soils. We then apply the new method to four surface soil samples in Fukushima to confirm the validity of the results.

\section{SAMPLING AND METHODS}

Sample Description. Figure 1 shows the Cs radioactivity distribution around the $\mathrm{FDNPP}^{32}$ and the sample locations for

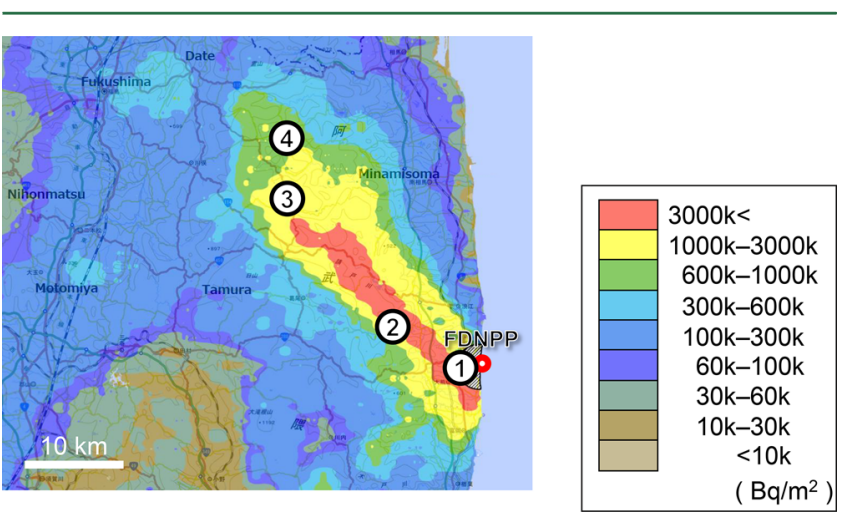

Figure 1. Distribution of $\mathrm{Cs}^{134+137}$ radioactivity and sample locations. The map was modified with permission from Extension Site of Distribution Map of Radiation Dose, etc./GSI Maps. ${ }^{32}$ The number represents the sampling points: no. 1, Ottozawa; no. 2, Omaru; no. 3, Nagadoro; and no. 4, Fukaya.

this study. During sampling campaigns in 2011 and 2012, surface soil samples in fields devoid of vegetation were collected from four locations in the Fukushima Prefecture: Ottozawa (no. 1), Omaru (no. 2), Nagadoro (no. 3), and Fukaya (no. 4), all being within a distance of $4.42-40.3 \mathrm{~km}$ from the FDNPP (Figure 1 and Table 1). These soil samples are mainly composed of typical soil minerals such as quartz, feldspars, 
Table 1. Information Concerning the Samples Used in the Present Study to Which the QCP Method Was Applied

\begin{tabular}{|c|c|c|c|c|c|}
\hline $\begin{array}{l}\text { sampling } \\
\text { point }\end{array}$ & $\begin{array}{l}\text { location } \\
\text { (sample number in KU sample archive) }\end{array}$ & latitude-longitude & $\begin{array}{l}\text { distance from } \\
\text { FDNPP }(\mathrm{km})\end{array}$ & soil type & sampling date \\
\hline no. 1 & Ottozawa (KU171) & $37^{\circ} 25^{\prime} 10.045^{\prime \prime} \mathrm{N}, 141^{\circ} 00^{\prime} 25.387^{\prime \prime} \mathrm{E}$ & 4.42 & $\begin{array}{l}\text { surface soil of } \\
\text { field }\end{array}$ & March 16, 2012 \\
\hline no. 2 & Omaru (KU1) & $37^{\circ} 28^{\prime} 00.490^{\prime \prime} \mathrm{N}, 140^{\circ} 55^{\prime} 47.514^{\prime \prime} \mathrm{E}$ & 13.0 & $\begin{array}{l}\text { surface soil of } \\
\text { field }\end{array}$ & December 20, 2012 \\
\hline no. 3 & Nagadoro (KU133) & $37^{\circ} 36^{\prime} 44.913^{\prime \prime} \mathrm{N}, 140^{\circ} 44^{\prime} 56.777^{\prime \prime} \mathrm{E}$ & 32.3 & $\begin{array}{l}\text { surface soil of } \\
\text { paddy }\end{array}$ & March 15, 2012 \\
\hline no. 4 & Fukaya (KU39) & $37^{\circ} 42^{\prime} 43.699^{\prime \prime} \mathrm{N}, 140^{\circ} 43^{\prime} 36.499^{\prime \prime} \mathrm{E}$ & 40.3 & $\begin{array}{l}\text { surface soil of } \\
\text { field }\end{array}$ & October 25,2012 \\
\hline
\end{tabular}

micas, and clay minerals. The details of soil characterization can be found in previous reports. $6,11,17,33$ The amount of adsorption sites in clay minerals is large compared to that of soluble radioactive Cs. The calibration and determination of the accuracy analysis of the autoradiography protocol were conducted using several radioactive particles: one Cs-clay, three gravels, and five CsMPs. The characteristics of these particles, including radioactivity, have already been reported in our previous study, ${ }^{22,23}$ and this is also summarized in Table 2.

Table 2. List of the Samples Used as Point Sources for the Calibration of the Radioactivity Measurement Using Autoradiography $^{a}$

\begin{tabular}{llll} 
sample name & $\begin{array}{c}\text { radioactivity } \\
(\mathrm{Bq})\end{array}$ & \multicolumn{1}{c}{ size (maximum diameter) } & type \\
NGD-clay1 & 0.228 & $0.50 \mathrm{~mm} \times 60 \mu \mathrm{m}$ height & Cs-clay \\
OTZ8 & 3.64 & $2.6 \mu \mathrm{m}$ & CsMP \\
OTZ9 & 4.89 & $2.0 \mu \mathrm{m}$ & CsMP \\
KOI2 & 17.8 & $4.4 \mu \mathrm{m}$ & CsMP \\
OTZ7 & 72.1 & $5.8 \mu \mathrm{m}$ & CsMP \\
G2 & 135 & $0.97 \mathrm{~mm}$ & gravel \\
G5 & 240 & $2.0 \mathrm{~mm}$ & gravel \\
G3 & 259 & $2.7 \mathrm{~mm}$ & gravel \\
OTZ3-before ${ }^{b}$ & 418 & $17 \mu \mathrm{m}$ & CsMP \\
OTZ3-after $^{b}$ & 334 & $17 \mu \mathrm{m}$ minus the size of the pit & CsMP \\
& & by the SIMS analysis &
\end{tabular}

${ }^{a}$ NGD-clayl and OTZ7 were used to draw the PSL intensity- $t$ diagram, and all samples were used to deduce the PSL/h-D graph. Detailed microscopic characteristics of some grains have been reported elsewhere. ${ }^{b}$ From Imoto and Ochiai et al. ${ }^{22}$ OTZ3-before and -after stand for the same CsMP before and after the SIMS analysis.

Safety. ${ }^{134} \mathrm{Cs}$ and ${ }^{137} \mathrm{Cs}$ are radioactive $\beta$-emitters with $\gamma$ emitting daughter isotopes. Radioisotopes should be handled by suitably qualified and experienced personnel in a properly equipped laboratory, and any work should follow appropriate risk assessment. The possession and use of radioactive materials is subject to statutory controls.

$\gamma$ Spectrometry. The ${ }^{134} \mathrm{Cs}$ and ${ }^{137} \mathrm{Cs}$ radioactivities of the individual CsMPs were determined using $\gamma$ spectrometry. Here, a point-source specimen, which was described in a previous study, ${ }^{11}$ was used for standardization. Briefly, the radioactivity of a microparticle with a size of $\sim 400 \mu \mathrm{m}$ obtained from the surface soil in Fukushima was precisely determined at the radioisotope center in Tsukuba University, Japan, and utilized as a standard point specimen for ${ }^{134} \mathrm{Cs}$ and ${ }^{137} \mathrm{Cs}$. The radioactivity of the point source standard was $23.9 \mathrm{~Bq}$ for ${ }^{134} \mathrm{Cs}$ and $94.6 \mathrm{~Bq}$ for ${ }^{137} \mathrm{Cs}$ as of September 29, 2015. The radioactivity measurements for the other particles and Cs clay were performed using SEIKO E\&G germanium semiconductor detectors GMX23, GMX30, and GMX40 at Kyushu Univer- sity's Radioisotope Center. The acquisition time ranged from 1 to $162 \mathrm{~h}$ to ensure that the net area of both the ${ }^{134} \mathrm{Cs}$ and ${ }^{137} \mathrm{Cs}$ peaks exceeded 1000 counts.

Quantification of CsMPs Method. A method to quantify the CsMPs, hereafter called the QCP method, was developed using the photostimulated luminescence (PSL) of an imaging plate (IP, FUJIFILM BAS-SR 2025) with an IP reader (GE, Typhoon FLA 5100). The pixel size was set to $50 \mu \mathrm{m}$. The PSL intensity is proportional to the radioactivity and exposure time. ${ }^{34}$ First, the relationship between the PSL intensity and the exposure time (herein PSL/h) was determined using two radioactive particles; a micron-sized clay particle that had adsorbed radioactive Cs (NGD-clay1) and a CsMP (OTZ7). These samples provided different total $\gamma$ radiation owing to their ${ }^{134} \mathrm{Cs}$ and ${ }^{137} \mathrm{Cs}$ content $(0.228 \pm 0.003$ and $72.1 \pm 0.7 \mathrm{~Bq}$ for NGD-clay1 and OTZ7, respectively). The exposure time varied from $15 \mathrm{~min}$ to $73 \mathrm{~h}$. The radioactivity of the standard microparticles was determined using $\gamma$ spectrometry.

Second, the relationship between the PSL intensity per hour and the radioactivity (in becquerel) was established by comparing six microparticles, one Cs-clay, and three large millimeter-sized gravels, whose radioactivities were previously determined to be different from each other using $\gamma$ spectrometry (Table 2). The exposure time for the PSL measurement was set such that the total PSL count became less than the upper limit, and a linear relation between the PSL intensity per hour and the radioactivity was retained.

Distinguishing between Cs-Clay and CsMPs by Setting a Threshold Radioactivity. A soil sample collected from Nagadoro was used to determine the threshold radioactivity for the CsMPs. First, $2.95 \mathrm{~g}$ of the soil sample was sieved with 597 and $114 \mu \mathrm{m}$ meshes in a fume hood and separated into three particle size fractions: $>597 \mu \mathrm{m}$ (large; $0.86 \mathrm{~g}$ ), $114-597 \mu \mathrm{m}$ (medium; $1.2 \mathrm{~g}$ ), and $<114 \mu \mathrm{m}$ (small; $0.88 \mathrm{~g}$ ). For the large-size fraction, radioactive particles were picked from the sample after autoradiography to isolate them. For the medium- and small-size fractions, the radioactive particles were separated according to the procedure for separating CsMPs from soil samples reported in our previous study. ${ }^{11}$ Briefly, the powder samples were dispersed on grid paper and covered with a plastic sheet, and an IP was then placed on the samples for 1 hour. After the positions of intensely radioactive spots were identified, droplets of pure water were added to these positions and then drawn using a pipet to produce suspensions with small amounts of soil particles by dilution with pure water. This procedure was repeated until the suspension did not contain a significant amount of soil particles. Subsequently, the isolated radioactive particles were characterized in detail to identify the Cs-clay, CsMPs, or other aggregates by means of the following procedures. (i) The Cs-clay was identified based on the platy 

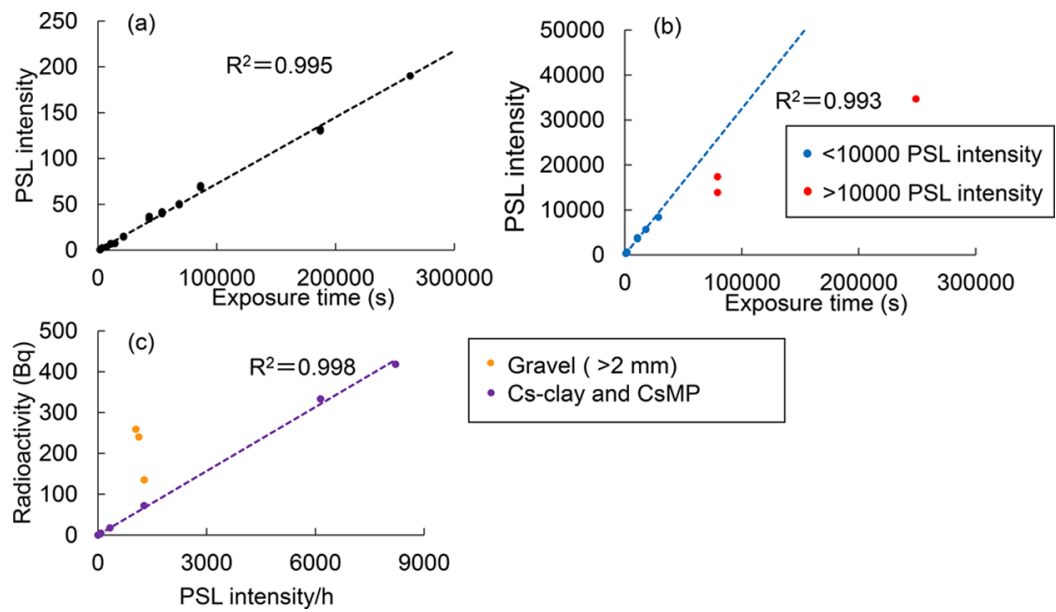

- Gravel ( $>2 \mathrm{~mm}$ )

- Cs-clay and CsMP

Figure 2. Correlation between parameters in the autoradiography analysis. (a) Correlation between the PSL intensity and the exposure time, which was measured using a Cs-clay (NGD-clay1). The regression line is shown as a dotted line. (b) Correlation between the PSL intensity and the exposure time, which was measured using a CsMP (OTZ7). The dotted line represents the regression line determined using the plots with $<10000$ PSL counts. (c) Correlation between the radioactivity and the PSL/h, based on measurements of one Cs-clay, six Cs MPs, and three gravels. The dotted line indicates the regression line created using the data for the Cs-clays and the CsMPs.

shape of micaceous minerals in the stereomicroscope image. (ii) To separate CsMPs that adhere to visible clay particles, the visible and radioactive clay particles were subjected to an ultrasonic treatment in ultrapure water for $20 \mathrm{~min}$, and then autoradiography was performed. It is noted that Cs in both clays and CsMPs scarcely dissociate in water during the sonication because it tightly binds to clay minerals ${ }^{33}$ or occurs within $\mathrm{SiO}_{2}$ glass matrix of CsMP. A visible clay particle was determined to be a Cs-clay if the radioactive spot remained at the position of the clay particle; however, it was considered to be a CsMP if the radioactive spot was dissociated from the clay particle. (iii) The radioactive particle was crushed using a blade, and autoradiography was then performed. The platy particle and aggregate were judged to be a Cs-clay and aggregate when the radioactive spot was separated along with the crushed fragments, ${ }^{28}$ whereas it was determined to be a CsMP if it remained as a single spot in the autoradiography image. (iv) The radioactive particles were found and characterized by scanning electron microscopy (Shimadzu, SS550 and Hitachi, SU6600) using an energy dispersive X-ray analysis (EDAX Genesis). Information on the shape and composition of the clay and aggregate were obtained. For the particular case, the CsMPs were also searched using SEM-EDX, although it was difficult to detect and characterize all of the CsMPs. The above four procedures were employed as appropriate for each sample.

After differences between CsMPs and Cs-clay were distinguished, a histogram of the particles was produced as a function of the radioactivity for the three size fractions (large, medium, and small) as described above. The radioactivity of each radioactive particle was calculated based on the QCP method described in the previous section. The threshold radioactivity was determined for the identification of the CsMPs using the histogram.

Application of the QCP Method to Surface Soils. Approximately $0.5 \mathrm{~g}$ of four soil samples from Ottozawa, Omaru, Nagadoro, and Fukaya, listed in Table 1, were subjected to ultrasonic treatment for $30 \mathrm{~min}$ and sieved with 597 and $114 \mu \mathrm{m}$ meshes. Next, the QCP method was applied. In the autoradiograph image of the small fraction, all spots were determined to be either Cs-clay, aggregate, or CsMP using the radioactivity threshold. The radioactivity fraction (RF) of the
CsMPs (i.e., the amount of radioactivity that can be attributed to the CsMPs) can be calculated using the following equation:

$$
\mathrm{RF}=\frac{D_{\mathrm{CsMP}}}{D_{\mathrm{all}}} \times 100(\%)
$$

Here, $D_{\text {CsMP }}$ is the sum of the CsMP radioactivity (in becquerel) in the soil samples determined by the QCP method, and $D_{\text {all }}$ represents the total radioactivity (in becquerel) of the soil sample determined by $\gamma$-ray spectrometry. The number of CsMPs in the soil samples was quantified by counting the radioactive spots greater than the radioactive threshold.

\section{RESULTS}

Correlation of the PSL Intensity and Radioactivity. Figure $2 \mathrm{a}$ shows the linear correlation between the PSL intensity and the exposure time for the measurement of the Csclay (NGD-clay1) with low radioactivity, $0.228 \pm 0.003 \mathrm{~Bq}$. A linear correlation between the PSL intensity and the exposure time was also obtained for the measurement of the CsMP (OTZ7) when the PSL intensity was less than 10000 (Figure $2 \mathrm{~b}$ ); however, the PSL plots deviated from the regression line of the correlation when the PSL intensity exceeded 10000 , which is approximately consistent with the maximum counts of the dynamic range of the imaging plate as certified by the manufacturer. Therefore, the upper limit of the PSL count was determined to be 10000 . For the correlation line between the PSL intensity and the exposure time, the standard deviation was calculated to be $\sigma=3.94$, which results in a limit of quantification for the PSL measurement of $10 \sigma=39.4$. In Figure $2 c$, the correlation between the PSL intensity per hour (PSL/h) and radioactivity (becquerel) is plotted for Cs -clays and CsMPs that have a small particle size (approximately the micron scale) and for gravel samples with a particle size of $>\sim 2$ $\mathrm{mm}$. The gravel sample data deviates from the regression line, which is attributed to the large geometric variation in the distance between the imaging plate and the samples. Based on the regression line excluding all the data for the gravel samples, a correlation of $0.0523( \pm 0.0008) \mathrm{Bq}$ for $1.00 \mathrm{PSL} / \mathrm{h}\left(R^{2}=\right.$ 0.998) was obtained; this was subsequently used for the 

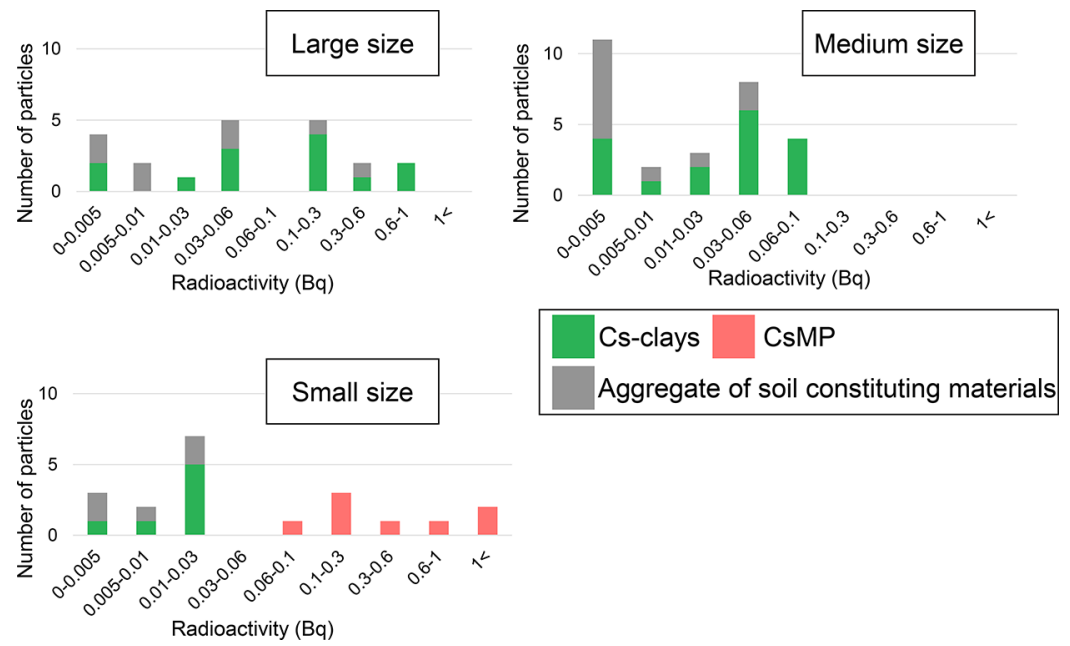
$<114 \mu \mathrm{m}$, respectively.

(a)

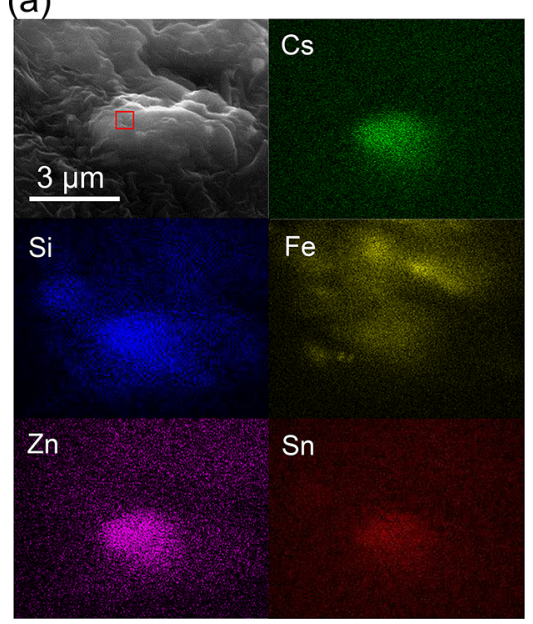

(b)

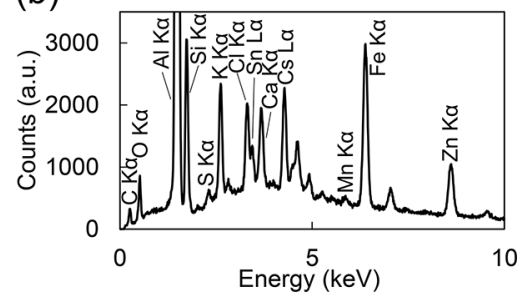

Figure 4. Scanning electron microscopy of the CsMP isolated from the Nagadoro soil sample. (a) Secondary electron (SE) image of a CsMP associated with the energy dispersive X-ray spectrum (EDX) elemental maps of the major constituents. (b) EDX spectrum of the area indicated by the red square in the SE image in panel a.

conversion of the PSL intensity to radioactivity in the following experiments. Note that the conversion factor is valid only when conditions are the same as the present experiment (i.e., FUJIFILM BAS-SR 2025 for the imaging plate, GE Typhoon FLA 5100 for the IP reader, and $50 \mu \mathrm{m}$ for the pixel size). When different experimental conditions are used, the parameters such as the pixel size, the exposure time, the maximum PSL count, and PSL intensity-radioactivity correlation need to be recalibrated.

Threshold Radioactivity for Distinguishing Cs-Clay and CsMPs. A total of 69 radioactive particles were isolated from the soil sample collected from Nagadoro and further investigated for their size, radioactivity, and whether they were CsMPs. We distinguished whether the isolated particles were CsMPs, Cs-clays, or aggregates based on the procedure described in the Sampling and Methods section. Based on the identification of all 69 particles, it was concluded that no CsMPs are present within the medium $(114-597 \mu \mathrm{m})$ and large $(>597 \mu \mathrm{m})$ size fractions, whereas CsMPs were identified in the small fraction $(<114 \mu \mathrm{m})$ with a radioactivity of $>0.06 \mathrm{~Bq}$ (Figure 3). Therefore, the criteria to identify CsMPs is that the particle is $<114 \mu \mathrm{m}$ with a radioactivity $>0.06 \mathrm{~Bq}$ for the present experimental setting. A CsMP was isolated from the fraction satisfying these criteria and characterized using a SEM-EDX (Figure 4). A secondary electron image shows an aggregate of the CsMP and the other soil particles attached on the double-sided carbon tape (Figure 4a). The composition of the CsMP is similar to the ones previously reported; ${ }^{11}$ its main constituents are oxides of $\mathrm{Si}, \mathrm{Fe}, \mathrm{Zn}, \mathrm{Sn}$, and Cs (Figure 4a,b), although the EDX spectrum has an $\mathrm{Al}$ signal derived from $\mathrm{Al}$ substrate and some other elements including $\mathrm{Ca}, \mathrm{K}$, and $\mathrm{Cl}$ as well as small interfering peaks from the constituents of clay and Fe oxide background. The ${ }^{134} \mathrm{Cs}$ and ${ }^{137} \mathrm{Cs}$ radioactivities of the CsMP were determined to be 1.90 and $1.85 \mathrm{~Bq}$, respectively (Table 3). The radioactivity was decay-corrected to March 12, 2011, 15:36 Japan standard time (JST), when the first hydrogen explosion occurred. The ${ }^{134} \mathrm{Cs}$-to- ${ }^{137} \mathrm{Cs}$ radioactivity ratio was $1.02 \pm 0.05$, confirming that the CsMP was released from the FDNPP. ${ }^{35}$

Application of the QCP Method to Surface-Soil Samples. Our newly developed QCP method was applied to four surface soil samples (Table 1). The autoradiography 
Table 3. Radioactivity of a CsMP Discovered in the Nagadoro Soil Sample ${ }^{a}$

\begin{tabular}{|c|c|c|c|}
\hline \multirow[b]{2}{*}{ particle size $(\mu \mathrm{m})$} & \multicolumn{2}{|c|}{ radioactivity $(\mathrm{Bq})^{b}$} & \multirow[b]{2}{*}{${ }^{134} \mathrm{Cs} /{ }^{137} \mathrm{Cs}$} \\
\hline & ${ }^{134} \mathrm{Cs}$ & ${ }^{137} \mathrm{Cs}$ & \\
\hline 1.18 & $1.90( \pm 0.10)$ & $1.85( \pm 0.01)$ & $1.02( \pm 0.05)$ \\
\hline
\end{tabular}

${ }^{a}$ The ${ }^{134} \mathrm{Cs}$ and ${ }^{137} \mathrm{Cs}$ radioactivities of an isolated individual CsMP measured using $\gamma$-spectrometry. ${ }^{b}$ The radioactivity was decaycorrected to March 12, 2011, 15:36 JST.

images for the three size fractions were collected using the QCP method for all four samples, and the images for a Nagadoro sample are shown in Figure 5 as representative data. The autoradiography images of the other samples are compiled in Figures S1-S3. Note that sieving with the 597 and $114 \mu \mathrm{m}$ meshes for the QCP method efficiently excluded the Cs-clay from the CsMP group. The pink arrows in the small fraction indicate the radioactive spots that satisfy the criteria described above and are identified as CsMPs (Figure 5). Each spot was manually counted and is indicated by an arrow. The sum of the radioactivity of all the spots was calculated to be $16.1 \pm 0.1 \mathrm{~Bq}$. The total radioactivity derived from the CsMP identified in the sample was used to calculate the RF of the CsMP. As a result, the RF of CsMPs in Nagadoro soil (as per Figure 5) was determined to be $19.8 \pm 0.3 \%$. The total number of radioactive spots identified as CsMPs was determined to be $154 \pm 4$ CsMPs per gram in the Nagadoro soil (see Table 3). The RF and the number of CsMPs in all four soil samples (Figures 5 and $\mathrm{S} 1-\mathrm{S} 3$ ) were determined to be $8.53-31.8 \%$ and $48-318$ CsMPs/g, respectively, indicating that the RF and the number varies greatly depending on the location (Table 4). In addition, the effect of sample volume on the QCP method was investigated using the soil with the lowest radioactivity, $\sim 6.8$ $\mathrm{Bq} / \mathrm{g}$, in which a limited number of CsMPs were present. The results of QCP method are summarized in Table S2. The radioactivity fractions of CsMPs were calculated to be $23 \%$ and $27 \%$ for 7 and $0.5 \mathrm{~g}$, respectively. The results obtained here are in good agreement with each other within the reasonable error range. Thus, the $0.5 \mathrm{~g}$ sample mass is a reasonably optimized mass for the application of the QCP method to the samples with total radioactivity as low as $\sim 7 \mathrm{~Bq} / \mathrm{g}$. Increasing the mass of the soils being analyzed would permit extension of the applicability of the technique to soils with radioactivities much lower than $\sim 7 \mathrm{~Bq} / \mathrm{g}$.

\section{DISCUSSION}

Validity of the Exclusion Procedure for Clay Particles Adsorbing Radioactive Cs. As mentioned above, the Cs concentration on the Cs-clay fraction is considered to be low; however, the radioactivity density of the clay has not been well evaluated. In Koarashi et al., ${ }^{36}$ assuming that all Cs is associated with clay fraction, the $\sim 60 \mathrm{kBq} / \mathrm{kg}$ maximum radioactivity corresponds to only $3-12 \mathrm{~Bq} / \mathrm{g}$ clay based on their volume percentage of $5-20 \%$. In contrast, another study ${ }^{37}$ reported that clay particles with a particle size of $\sim 50 \mu \mathrm{m}$ can sorb Cs as much as $10^{-2} \mathrm{~Bq}^{37}$ Thus, there is a possibility that the highest Cs radioactivity on clay could exceed the threshold value. To confirm and determine the applicability of the QCP method, we have isolated radioactive particles from the Ottozawa soil, and the most-radioactive clay particle was isolated for further SEM observation (Figure S4). The volume of this particle was approximated based on the two SEM images tilted at $0^{\circ}$ and $45^{\circ}$. The composition was qualitatively analyzed by SEM-EDX and was similar to that of weathered biotite. The radioactivity was determined to be $0.514 \pm 0.013 \mathrm{~Bq}$ using $\gamma$ spectroscopy. The radioactivity density was calculated to be $\sim 1.3 \times 10^{4} \mathrm{~Bq} / \mathrm{g}$ assuming the density of clay as $2.8 \mathrm{~g} / \mathrm{cm}^{3}$. Here we assume that the radioactivity density can be applied to micron-sized clay particles in the present study because Mukai et al. reported that radioactive $\mathrm{Cs}$ is uniformly distributed on weathered biotite particles as small as $\sim 100 \mu \mathrm{m}$ that were collected from Fukushima. ${ }^{28}$ Based on this calculated value, the validity of the method distinguishing between CsMPs and Cs-clays is discussed below.

The present analytical method determined the criteria for finding CsMPs: smaller than $114 \mu \mathrm{m}$ and a threshold radioactivity of $0.06 \mathrm{~Bq}$, which was the lower limit of the range of radioactivity for CsMPs in the frequency histogram. Here, assuming a Cs-clay particle that has the maximum radioactivity and is $114 \mu \mathrm{m}$ in size, the radioactivity of a Cs-clay (D) can be calculated as follows:

$$
D=D_{\mathrm{d}} \times d \times V
$$

where $D_{\mathrm{d}}$ is the highest radioactive density of Cs-clay determined above $\left(\sim 1.3 \times 10^{4} \mathrm{~Bq} / \mathrm{g}\right), d$ is the mass density of Cs-clay (in which the typical density of clay minerals, $\sim 2.8$ $\mathrm{g} / \mathrm{cm}^{3}$, is adopted), ${ }^{38}$ and $V$ is the largest volume of Cs-clay that can pass through a $114 \mu \mathrm{m}$ mesh. To calculate the volume, a hexagonal polyhedron with a longest diameter of $114 \mu \mathrm{m}$ (the length of one side is $57 \mu \mathrm{m}$ ) with a height of $114 \mu \mathrm{m}$ was considered, where the volume was calculated to be $\sim 1 \times 10^{-6}$ $\mathrm{cm}^{3}$. This volume gave a maximum possible radioactivity of an
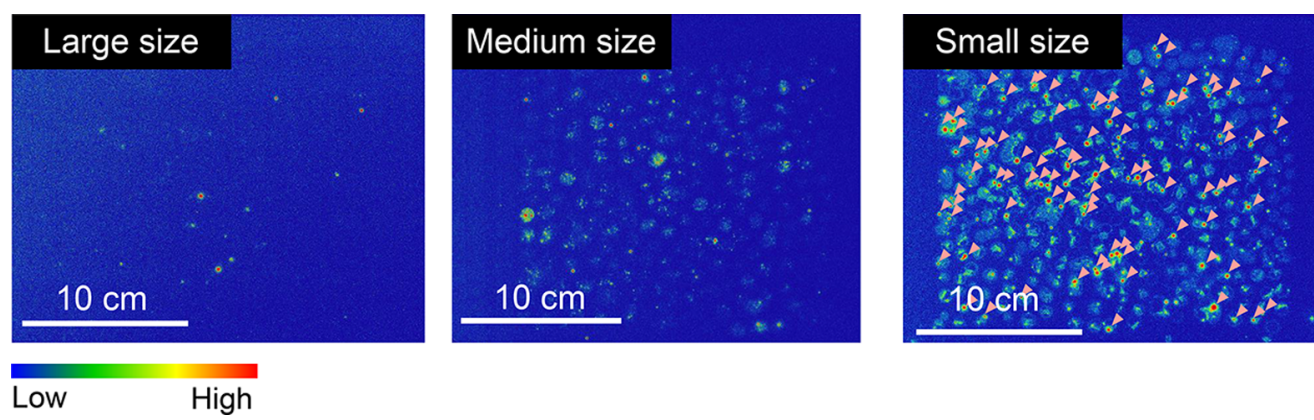

Low

Figure 5. Representative result of the QCP method for the Nagadoro soil sample. Color-scaled autoradiography images of the large, medium, and small size fractions indicating the sizes of the particles ( $>597,114-597$, and $<114 \mu$ m, respectively). The pink arrows indicate the CsMP spots, where the radioactivity was determined to be higher than the threshold value $(0.06 \mathrm{~Bq})$. The exposure time was 5 days. 
Table 4. Results of the QCP Method Applied to Soil Samples from Ottozawa, Omaru, Nagadoro, and Fukaya

\begin{tabular}{lcccc}
\multicolumn{1}{c}{ area } & $\begin{array}{c}\text { mass } \\
(\mathrm{g})\end{array}$ & $\begin{array}{c}\text { number of CsMPs } \\
(\text { particles per gram })^{a}\end{array}$ & $\begin{array}{c}\text { total radioactivity } \\
(\mathrm{Bq} / \mathrm{g})^{b}\end{array}$ & $\begin{array}{c}\text { total radioactivity of CsMPs } \\
(\mathrm{Bq} / \mathrm{g})^{a}\end{array}$ \\
Ottozawa & 0.453 & $318( \pm 18)$ & $1016( \pm 10)$ & $86.8( \pm 1.1)$ \\
Omaru & 0.532 & $165( \pm 5)$ & $236( \pm 1)$ & $44.9( \pm 0.3)$ \\
Nagadoro & 0.539 & $154( \pm 4)$ & $151( \pm 2)$ & $29.9( \pm 0.3)$ \\
Fukaya & 0.554 & $48.2( \pm 3.7)$ & $46.6( \pm 0.2)$ & $14.8( \pm 0.2)$
\end{tabular}

${ }^{a}$ The number and total radioactivity of the CsMPs were measured using the QCP method. ${ }^{b}$ The total radioactivity of the soil samples was analyzed via $\gamma$-spectrometry.

individual Cs-clay of $D=\sim 0.036 \mathrm{~Bq}$, which is lower than the threshold radioactivity of a CsMP, $0.06 \mathrm{~Bq}$. This calculation clearly indicates the validity of this procedure for distinguishing between CsMPs and Cs-clay in the present experiment. Thus, it is assured that the QCP method is applicable to the soils contaminated with radioactive $\mathrm{Cs}$, of which the bulk radioactivity is as high as that of the Ottozawa soil, $10^{3} \mathrm{~Bq} / \mathrm{g}=10^{6}$ $\mathrm{Bq} / \mathrm{kg}$ (Table 4). However, it is plausible that the soil samples that have greater total radioactivity than that of Ottozawa contain clay particles with a radioactivity density higher than the threshold value of CsMP. Thus, the QCP method can be applicable to the soil with total Cs radioactivity of $<10^{6} \mathrm{~Bq} / \mathrm{kg}$, which actually covers most of the area of the Fukushima prefecture. It cannot be applicable only to soil samples with total Cs radioactivity concentration (hot spot) near the FDNPP. Thus, the merit of QCP method still stands, and it is important for the quantification of CsMPs in ambient environments.

Furthermore, the procedure of the present QCP method can be easily optimized for the application to the soils with higher total radioactivity than $10^{6} \mathrm{~Bq} / \mathrm{kg}$. Using the sieving mesh with smaller pore size than $114 \mu \mathrm{m}$ (e.g., a $10 \mu \mathrm{m}$ sieve) would exclude the presence of Cs-clay with higher radioactivity than the threshold value of CsMP. Thus, the modified QCP can be used to quantify the number of CsMPs in sample regardless of the total radioactivity.

Evaluation of the Particle Diameter of CsMPs that Correspond to the Threshold Radioactivity. The threshold radioactivity determined in the present study is the minimum radioactivity of CsMPs that can be separated from the soil; however, there is still ambiguity if the threshold corresponds to a reasonable size of the CsMPs. Therefore, the diameter of a particle having the threshold radioactivity was estimated as follows.

Assuming the shape of CsMP as sphere, the particle diameter of a CsMP having the threshold radioactivity $\left(\Phi_{\text {th }}\right)$ can be expressed as:

$$
\begin{aligned}
& \Phi_{\mathrm{th}}=2\left(\frac{3 V}{4 \pi}\right)^{1 / 3} \\
& V=\frac{D_{\mathrm{th}}}{D_{\mathrm{d}, \text { max }} d \times 10^{12}}
\end{aligned}
$$

where $D_{\text {th }}$ is the threshold radioactivity of a CsMP (0.06 Bq); $D_{\mathrm{d} \text {,max }}$ is the maximum radioactivity density of the CsMPs that have been characterized so far $\left(7.77 \pm 0.09 \times 10^{11} \mathrm{~Bq} / \mathrm{g}\right)$; $d$ is the density of the CsMPs, which was set to $2.65 \mathrm{~g} / \mathrm{cm}^{3}$ based on the similar chemical properties of $\mathrm{SiO}_{2}$ glass; ${ }^{39} \mathrm{~V}$ is the volume of the CsMP; and $\Phi_{t h}$ is the diameter of the CsMP with the threshold radioactivity. The calculation resulted in $0.4 \mu \mathrm{m}$ for $\Phi_{\mathrm{th}}$.
Figure 6 illustrates the distribution of the radioactivity of CsMPs that have $<5 \mathrm{~Bq}$ as a function of the particle size, as

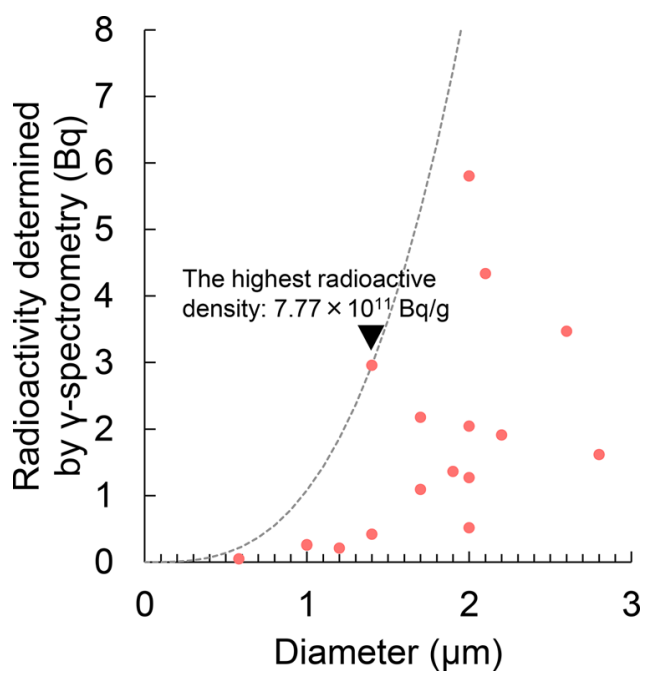

Figure 6. Radioactivity of CsMPs as a function of their size. A summary of the radioactivities of CsMPs as a function of their particle size for the particles that have been discovered to date.,11,22,23 The dotted curve indicates the ideal relation between the radioactivity and the size for CsMPs having the maximum radioactivity density, assuming a homogeneous radioactivity density within the particle.

reported by previous studies. ${ }^{9,11,22}$ As can been seen in the diagram, the smallest CsMP reported so far is $0.58 \mu \mathrm{m}$ in diameter, ${ }^{9}$ which is larger than $\Phi_{\text {th. }}$. This result may indicate low probability of finding CsMPs $<\sim 0.5 \mu \mathrm{m}$ in the environment. The histogram of the particle's radioactivity in Figure 3 reveals that the distribution of CsMPs is clearly separated from that of Cs-clay particles, and there are no CsMPs with radioactivity less than $0.06 \mathrm{~Bq}$. Therefore, it is likely that CsMPs released into the environment are generally larger than $\sim 0.5 \mu \mathrm{m}$. This hypothesis is partly supported by a previous report by the Nuclear Energy Agency, ${ }^{40}$ which theoretically calculated the aggregation of particles smaller than $0.1 \mu \mathrm{m}$ during the molten core-concrete interactions in a severe accident. A previous experimental study of molten core-concrete interactions also revealed the formation of microparticles as aggregates of $0.1 \mu \mathrm{m}$ sized clusters. ${ }^{41}$ Indeed, previous transmission electron microscopy images revealed evidence of aggregation within a single particle..$^{22,23}$ It is noted that in the presented method, aggregates that cannot be separated by the sonication process would be considered a single particle.

Implications of the QCP Method for Evaluating CsMPs in the Biosphere. The QCP method developed in the present study is a simple but powerful method of quantifying the amount of radioactivity and number of CsMPs in soil samples, even though the threshold values need to be reset depending 
on the analytical protocol, such as the IP and the IP reader. The RF value of the CsMPs was determined to be as high as $32 \%$ in one soil sample, proving the significant contribution of CsMPs to the total radioactivity in the FDNPP-affected environment. The number of soil samples analyzed in the present study was too limited to draw a detailed quantitative picture of the CsMP distribution. Constructing a map of the CsMP distribution by analyzing a larger set of samples would provide quantitative insight into understanding the timing of the formation, release, and movement of CsMPs from the FDNPP during the initial stage of the nuclear disaster. Due to their highly localized radioactivity, the quantity of CsMPs has been of great concern in residential areas in the Fukushima Prefecture. The RF values determined using the QCP method can be used as a representative indicator to convert the total Cs radioactivity to the numbers of CsMPs in each residential area near the data points. In addition, the contribution of CsMPs to the migration of radioactive $\mathrm{Cs}$ in the environment after the nuclear disaster has largely been overlooked as are their potential role in human metabolism. Although it is difficult to directly link the RF value and the number of CsMPs with the health impacts, those data are essential for the subsequent calculation of the radiation exposure. The number of the CsMPs is a measure of potential resuspension to air. Some fraction of the resuspended CsMPs can potentially be deposited deep in the respiratory system. ${ }^{9,42}$

It is important to predict the redistribution of the radiation dose over decadal time periods considering CsMPs. Indeed, Cs migration in the form of CsMPs is another important route for Cs transport in the environment, which can be tracked using the QCP method. Simple distribution coefficient, $K_{\mathrm{d}}$ or surfacecomplexation models will not be useful for the description of the migration of this form of Cs in the environment, in contrast to the ionic forms of Cs.

\section{ASSOCIATED CONTENT}

\section{S Supporting Information}

The Supporting Information is available free of charge on the ACS Publications website at DOI: 10.1021/acs.est.7b06693.

Tables showing radioactivity of the individual particles and results of the QCP method. Figures showing the results of the QCP method and secondary-electron images and elemental maps. (PDF)

\section{AUTHOR INFORMATION}

\section{Corresponding Author}

*E-mail: utsunomiya.satoshi.998@m.kyushu-u.ac.jp.

\section{ORCID}

Gareth T. W. Law: 0000-0002-2320-6330

Satoshi Utsunomiya: 0000-0002-6197-9705

\section{Author Contributions}

S.U. conceived the idea and designed all experiments. R.I. conducted QCP analysis. R.I. and S.U. wrote the manuscript. A.O., M.S., T.K., and G.F. performed the separation of CsMPs and SEM analysis. T.O. provided navigation during field research in Fukushima. S.Y. performed $\gamma$ spectroscopy at Tsukuba University. W.R.B., G.T.W.L, B.G., and R.C.E. participated in the discussion and interpretation of the results.

\section{Notes}

The authors declare no competing financial interest.

\section{ACKNOWLEDGMENTS}

The authors are grateful to Dr. Watanabe for her assistance on SEM analyses at the Center of Advanced Instrumental Analysis, Kyushu University. This study is partially supported by JST Initiatives for Atomic Energy Basic and Generic Strategic Research and by a Grant-in-Aid for Scientific Research (KAKENHI) from the Japan Society for the Promotion of Science (grant nos. 16K12585, 16H04634, and JP26257402). S.U. is also supported by The Mitsubishi Foundation/Research Grants in the Natural Sciences and by ESPEC Foundation for Global Environment Research and Technology (Charitable Trust) (ESPEC Prize for the Encouragement of Environmental Studies). G.T.W.L and W.R.B acknowledge RCUK funding (grant nos. NE/M014088/1 and ST/N002474/1). The findings and conclusions of the authors of this paper do not necessarily state or reflect those of the JST.

\section{REFERENCES}

(1) International Atomic Energy Agency. The Fukushima Daiichi Accident - Report by the Director General; International Atomic Energy Agency: Vienna, Austria, 2015.

(2) Steinhauser, G.; Brandl, A.; Johnson, T. E. Comparison of the Chernobyl and Fukushima nuclear accidents: A review of the environmental impacts. Sci. Total Environ. 2014, 470-471, 800-817.

(3) Koo, Y.-H.; Yang, Y.-S.; Song, K.-W. Radioactivity release from the Fukushima accident and its consequences: A review. Prog. Nucl. Energy 2014, 74, 61-70.

(4) Chino, M.; Nakayama, H.; Nagai, H.; Terada, H.; Katata, G.; Yamazawa, H. Preliminary Estimation of Release Amounts of 131 I and 137 Cs Accidentally Discharged from the Fukushima Daiichi Nuclear Power Plant into the Atmosphere. J. Nucl. Sci. Technol. 2011, 48 (7), 1129-1134.

(5) Buesseler, K.; Dai, M.; Aoyama, M.; Benitez-Nelson, C.; Charmasson, S.; Higley, K.; Maderich, V.; Masqué, P.; Morris, P. J.; Oughton, D.; et al. Fukushima Daiichi-derived radionuclides in the ocean: transport, fate, and impacts. Annu. Rev. Mar. Sci. 2017, 9 (1), $173-203$

(6) Kaneko, M.; Iwata, H.; Shiotsu, H.; Masaki, S.; Kawamoto, Y.; Yamasaki, S.; Nakamatsu, Y.; Imoto, J.; Furuki, G.; Ochiai, A.; et al. Radioactive Cs in the Severely Contaminated Soils Near the Fukushima Daiichi Nuclear Power Plant. Front. Energy Res. 2015, 3, 37.

(7) Nakajima, T.; Misawa, S.; Morino, Y.; Tsuruta, H.; Goto, D.; Uchida, J.; Takemura, T.; Ohara, T.; Oura, Y.; Ebihara, M.; et al. Model depiction of the atmospheric flows of radioactive cesium emitted from the Fukushima Daiichi Nuclear Power Station accident. Prog. Earth Planet. Sci. 2017, 4 (1), 2.

(8) Morino, Y.; Ohara, T.; Nishizawa, M. Atmospheric behavior, deposition, and budget of radioactive materials from the Fukushima Daiichi nuclear power plant in March 2011. Geophys. Res. Lett. 2011, 38 (7), 1.

(9) Imoto, J.; Furuki, G.; Ochiai, A.; Yamasaki, S.; Nanba, K.; Ohnuki, T.; Grambow, B.; Ewing, R. C.; Utsunomiya, S. Caesium fallout in Tokyo on 15th March, 2011 is dominated by highly radioactive, caesium-rich microparticles. Sci. Rep. 2017, 7, 42731, in press.

(10) Tsuruta, H.; Oura, Y.; Ebihara, M.; Ohara, T.; Nakajima, T. First retrieval of hourly atmospheric radionuclides just after the Fukushima accident by analyzing filter-tapes of operational air pollution monitoring stations. Sci. Rep. 2015, 4 (1), 6717.

(11) Furuki, G.; Imoto, J.; Ochiai, A.; Yamasaki, S.; Nanba, K.; Ohnuki, T.; Grambow, B.; Ewing, R. C.; Utsunomiya, S. Caesium-rich micro-particles: A window into the meltdown events at the Fukushima Daiichi Nuclear Power Plant. Sci. Rep. 2017, 7, 42731.

(12) Brockmann, J. E. Ex-vessel releases: Aerosol source terms in reactor accidents. Prog. Nucl. Energy 1987, 19 (1), 7-68. 
(13) Devell, L.; Johansson, K. Specific Features of Cesium Chemistry and Physics Affecting Reactor Accident Source Term Predictions; OECD/ NEA: Stockholm, Sweden, 1994.

(14) Auvinen, A.; Lehtinen, K. E. J.; Enriquez, J.; Jokiniemi, J. K.; Zilliacus, R. Vaporisation rates of $\mathrm{CsOH}$ and CsI in conditions simulating a severe nuclear accident. J. Aerosol Sci. 2000, 31 (9), 10291043.

(15) Kaneyasu, N.; Ohashi, H.; Suzuki, F.; Okuda, T.; Ikemori, F. Sulfate aerosol as a potential transport medium of radiocesium from the Fukushima nuclear accident. Environ. Sci. Technol. 2012, 46 (11), $5720-5726$.

(16) Kinoshita, N.; Sueki, K.; Sasa, K.; Kitagawa, J. -i.; Ikarashi, S.; Nishimura, T.; Wong, Y.-S.; Satou, Y.; Handa, K.; Takahashi, T.; et al. Assessment of individual radionuclide distributions from the Fukushima nuclear accident covering central-east Japan. Proc. Natl. Acad. Sci. U. S. A. 2011, 108 (49), 19526-19529.

(17) Iwata, H.; Shiotsu, H.; Kaneko, M.; Utsunomiya, S. Nuclear Accidents inFukushima, Japan, and Exploration of Effictive Decontaminant for the ${ }^{137} \mathrm{Cs}$-Contaminated Soils. In Advances in Nuclear Fuel; Revankar, S., Ed.; Intech: London, England, 2012; pp 123-174. (18) McKinley, J. P.; Zachara, J. M.; Heald, S. M.; Dohnalkova, A.; Newville, M. G.; Sutton, S. R. Microscale Distribution of Cesium Sorbed to Biotite and Muscovite. Environ. Sci. Technol. 2004, 38 (4), 1017-1023.

(19) Cremers, A.; Elsen, A.; De Preter, P.; Maes, A. Quantitative analysis of radiocaesium retention in soils. Nature 1988, 335 (6187), 247-249.

(20) Comans, R. N. J.; Hockley, D. E. Kinetics of cesium sorption on Illite. Geochim. Cosmochim. Acta 1992, 56 (3), 1157-1164.

(21) Fuller, A. J.; Shaw, S.; Ward, M. B.; Haigh, S. J.; Mosselmans, J. F. W.; Peacock, C. L.; Stackhouse, S.; Dent, A. J.; Trivedi, D.; Burke, I. T. Caesium incorporation and retention in Illite interlayers. Appl. Clay Sci. 2015, 108, 128-134.

(22) Imoto, J.; Ochiai, A.; Furuki, G.; Suetake, M.; Ikehara, R.; Horie, K.; Takehara, M.; Yamasaki, S.; Nanba, K.; Ohnuki, T.; et al. Isotopic signature and nano-texture of cesium-rich micro-particles: Release of uranium and fission products from the Fukushima Daiichi Nuclear Power Plant. Sci. Rep. 2017, 7 (1), 5409.

(23) Ochiai, A.; Imoto, J.; Suetake, M.; Komiya, T.; Furuki, G.; Ikehara, R.; Yamasaki, S.; Law, G. T. W.; Ohnuki, T.; Grambow, B.; et al. Nuclear fuel fragments released to the environment with cesiumrich microparticles from the Fukushima Daiichi Nuclear Power Plant. Environ. Sci. Technol. 2018, 52 (5), 2586-2594.

(24) Satou, Y.; Sueki, K.; Sasa, K.; Yoshikawa, H.; Nakama, S.; Minowa, H.; Abe, Y.; Nakai, I.; Ono, T.; Adachi, K.; et al. Analysis of two forms of radioactive particles emitted during the early stages of the Fukushima Dai-ichi Nuclear Power Station accident. Geochem. J. 2018, 52, 137-143.

(25) Kaltofen, M.; Gundersen, A. Radioactively-hot particles detected in dusts and soils from Northern Japan by combination of gamma spectrometry, autoradiography, and SEM/EDS analysis and implications in radiation risk assessment. Sci. Total Environ. 2017, 607-608, $1065-1072$.

(26) Tanaka, K.; Sakaguchi, A.; Kanai, Y.; Tsuruta, H.; Shinohara, A.; Takahashi, Y. Heterogeneous distribution of radiocesium in aerosols, soil and particulate matters emitted by the Fukushima Daiichi Nuclear Power Plant accident: retention of micro-scale heterogeneity during the migration of radiocesium from the air into ground and rive. $J$. Radioanal. Nucl. Chem. 2013, 295 (3), 1927-1937.

(27) Niimura, N.; Kikuchi, K.; Tuyen, N. D.; Komatsuzaki, M.; Motohashi, Y. Physical properties, structure, and shape of radioactive Cs from the Fukushima Daiichi Nuclear Power Plant accident derived from soil, bamboo and shiitake mushroom measurements. J. Environ. Radioact. 2015, 139, 234-239.

(28) Mukai, H.; Hatta, T.; Kitazawa, H.; Yamada, H.; Yaita, T.; Kogure, T. Speciation of Radioactive Soil Particles in the Fukushima Contaminated Area by IP Autoradiography and Microanalyses. Environ. Sci. Technol. 2014, 48 (22), 13053-13059.
(29) Kushin, V. V.; Lyscov, V. N. Quantitative track autoradiography of hot particles produced by the chernobyl accident. Nucl. Tracks Radiat. Meas. 1993, 21 (2), 277-282.

(30) Zeissler, C. J.; Forsley, L. P. G.; Lindstrom, R. M.; Newsome, S.; Kirk, A.; Mosier-Boss, P. A. Radio-microanalytical particle measurements method and application to Fukushima aerosols collected in Japan. J. Radioanal. Nucl. Chem. 2013, 296 (2), 1079-1084.

(31) Itoh, S.; Eguchi, T.; Kato, N.; Takahashi, S. Radioactive particles in soil, plant, and dust samples after the Fukushima nuclear accident. Soil Sci. Plant Nutr. 2014, 60 (4), 540-550.

(32) The Ministry of Education, Culture, Sports, Science and Technology (MEXT) and the Geospatial Information Authority of Japan (GSI). About the Establishment of the Extension Site of Distribution Map of Radiation Dose, Etc. http://ramap.jmc.or.jp/ map/ (accessed Dec 6, 2017).

(33) Yamasaki, S.; Imoto, J.; Furuki, G.; Ochiai, A.; Ohnuki, T.; Sueki, K.; Nanba, K.; Ewing, R. C.; Utsunomiya, S. Radioactive Cs in the estuary sediments near Fukushima Daiichi Nuclear Power Plant. Sci. Total Environ. 2016, 551-552, 155-162.

(34) Zeissler, C. J.; Lindstrom, R. M.; McKinley, J. P. Radioactive particle analysis by digital autoradiography. J. Radioanal. Nucl. Chem. 2001, 248 (2), 407-412.

(35) Nishihara, K.; Iwamoto, H.; Suyama, K. Estimation of fuel compositions in Fukushima-Daiichi Nuclear Power Plant; JAEA-Data/ Code 2012-018, Japan Atomic Energy Agency: Ibaraki, Japan, 2012.

(36) Koarashi, J.; Atarashi-Andoh, M.; Matsunaga, T.; Sato, T.; Nagao, S.; Nagai, H. Factors affecting vertical distribution of Fukushima accident-derived radiocesium in soil under different landuse conditions. Sci. Total Environ. 2012, 431, 392-401.

(37) Motai, S.; Mukai, H.; Watanuki, T.; Ohwada, K.; Fukuda, T.; Machida, A.; Kuramata, C.; Kikuchi, R.; Yaita, T.; Kogure, T. Mineralogical characterization of radioactive particles from Fukushima soil using $\mu$-XRD with synchrotron radiation. J. Mineral. Petrol. Sci. 2016, 111 (5), 305-312.

(38) Osipov, V. I. Density of clay minerals. Soil Mech. Found. Eng. 2012, 48 (6), 231-240.

(39) CRC Handbook of Chemistry and Physics, 95th ed.; Haynes, W. M., Lide, D. R., Bruno, T. J., Eds.; CRC Press: Boca Raton, FL, 2014.

(40) Nuclear Energy Agency. State-of-the-art report on nuclear aerosols; NEA/CSNI/R(2009)5; OECD/NEA: Boulogne-Billancourt, France, 2009, 388 pp.

(41) Kissane, M. P. On the nature of aerosols produced during a severe accident of a water-cooled nuclear reactor. Nucl. Eng. Des. 2008, 238 (10), 2792-2800.

(42) Schlesinger, R. B. Deposition and clearance of inhaled particles. In Concepts in inhalation toxicology; McClellan, R. O., Henderson, R. F., Eds.; Hemisphere: New York, 1989; pp 163-192. 